\title{
Monitoring the Effective Sterilization of Low-Temperature Hydrogen Peroxide Gas Plasma Sterilizers in 58 Hospitals - 22 PLADs, China, June 2015-December 2019
}

Jiaqi Wang'; Baoying Zhang'; Huihui Sun'; Jian Zhang'; Hongyang Duan ${ }^{1}$; Haiqun Ban'; Jin Shen ${ }^{1, *}$; Liubo Zhang'

\begin{abstract}
Summary
What is already known on this topic?

Hydrogen peroxide sterilizeation is widely used for luminal devices. However, the low penetrability of the sterilant is of major concern.

What is added by this report?

This report investigated the effective sterilization of low-temperature hydrogen peroxide gas plasma sterilizers and compared the applicability of different biological monitoring methods based on medical luminal devices.

What are the implications for public health practice?

It is recommended to use a biological process challenge device for monitoring the sterilization of luminal devices with low-temperature hydrogen peroxide gas plasma sterilizers.
\end{abstract}

With minimally invasive techniques improving, various types of medical luminal devices are widely used in surgeries. Low-temperature hydrogen peroxide gas plasma sterilizers significantly shorten the sterilization cycle and greatly improve the turnover rate of endoscopic instruments. This study aimed to investigate the effective sterilization of low-temperature hydrogen peroxide gas plasma sterilizers and to explore the applicability of different biological monitoring methods based on medical luminal devices. Basic information of low-temperature hydrogen peroxide gas plasma sterilizers in 58 hospitals across 22 provinciallevel administrative divisions (PLADs) was investigated, and these sterilizers were tested with biological process challenge devices (PCD) and normal biological indicators $(\mathrm{BI})$ at the same time to test the effectiveness of sterilization and to compare the monitoring results. The qualification rate, i.e., that the sterilizing process was effective, of biological PCD monitoring was lower than that of common biological monitoring. Therefore, more effective personnel training should be employed for the use of hydrogen peroxide gas plasma sterilizers. In addition, use of biological PCD monitoring when sterilizing tubetype/luminal devices is recommended.

Central sterile supply departments, operating rooms, and endoscopy centers in most of the hospitals are equipped with low-temperature hydrogen peroxide gas plasma sterilizers. However, due to the long lumen and the often-complex structures of luminal devices, they are often improperly sterilized. To understand the effective sterilization of luminal devices sterilized by low-temperature hydrogen peroxide gas plasma sterilizers, a survey was carried out as part of "National Surveillance Program for Hospital Disinfection and Sterilization Effects and Nosocomial Infection" conducted by China CDC. This survey included 58 sentinel hospitals across 22 PLADs of China.

A questionnaire was designed to investigate the lowtemperature hydrogen peroxide gas plasma sterilizers, including items needing to be sterilized, and biological monitoring methods, etc.

PCD biological indicators used in this study, which contain a self-contained bacterial tablet with recovery medium and 2 thin plastic tubes, the inner diameter of 1 millimeter and length of 1,000 millimeters, and common self-contained biological indicators were issued to the sentinel hospitals, where the indicating strain was Bacillus stearothermophilus ATCC7953. From June 2015 to December 2019, the investigators from provincial and municipal-level CDCs conducted the examination of sterilizers in the surveyed hospitals.

The following biological monitoring methods were applied: 1) the common biological indicator and PCD biological indicator were placed close to the cabinet door near the lower shelf inside the sterilization chamber and were sterilized with other objects; 2) after a complete sterilization cycle, the two kinds of biological indicators were inoculated in a $56{ }^{\circ} \mathrm{C}$ incubator with positive controls and cultured for 48 hours. A positive control culture being observed with a 
negative test group culture was judged as qualified sterilization, while a positive control culture with a positive test group culture was judged as unqualified sterilization. Statistical analysis was performed using SPSS for Windows (version 19, SPSS Inc., Chicago, Il, USA). The chi-squared test was used to compare the results of biological PCD monitoring with those of general biological monitoring. A $P$-value of $<0.05$ was considered to be statistically significant.

A total of 114 questionnaires were collected from 58 sentinel hospitals. Survey results showed that sterilizer use life ranged from 0.5 to 14 years, while about $24.6 \%$ of the sterilizers were over 10 years old. In this survey, laparoscopy, cystoscope, arthroscopy, choledochoscope, hysteroscope, and similar luminal devices were included, which implied sterilization by hydrogen peroxide. The tubes for these luminal devices have the smallest diameter of only 1 millimeter and a length of 50-130 centimeters. Low-temperature hydrogen peroxide gas plasma sterilizers are primarily used in central sterile supply departments $(63.2 \%$, $72 / 114)$, operating rooms $(34.2 \%, 39 / 114)$, and endoscopy centers $(2.6 \%, 3 / 114)$ (Table 1). All sterilizers are used for sterilization of luminal devices, such as endoscopies. In the survey, 38 sterilizers $(33.3 \%)$ used biological PCD in daily monitoring, and the remaining 76 sterilizers still used common biological indicators for luminal devices.

Biological PCD monitoring and common biological monitoring were simultaneously conducted 338 times. The qualification rate of biological PCD monitoring was $95.6 \%$ (323/338), while common biological monitoring was $99.1 \%$ (335/338); the difference was statistically significant $\quad(P<0.001, \quad$ Fisher exact probability test) (Table 2$)$.

\section{DISCUSSION}

According to survey results, low-temperature hydrogen peroxide gas plasma sterilizers are widely used for various types of lumen devices. Common biological indicators are designed to monitor effective sterilization in non-lumen medical devices but cannot effectively monitor effective sterilization in devices with long lumens. In addition, the lumen's length and the material to be sterilized also affect the sterilization effect (1). The PCD is defined according to American national standard AAMI TIR31 as an item designed to simulate a product to be sterilized, to constitute a determined challenge to the sterilization process, and
TABLE 1. Biological monitoring of low-temperature hydrogen peroxide sterilizers in 58 sentinel hospitals across 22 PLADs from June 2015 to December 2019.

\begin{tabular}{ccccc}
\hline $\begin{array}{c}\text { Hospital } \\
\text { grade }\end{array}$ & Department & $\begin{array}{c}\text { Biological } \\
\text { PCD }\end{array}$ & $\begin{array}{c}\text { Common } \\
\text { BI }\end{array}$ & Total \\
\hline Secondary & CSSD & 3 & 11 & 14 \\
& Operating room & 3 & 8 & 11 \\
Tertiary & CSSD & 23 & 35 & 58 \\
& Endoscopic Center & 1 & 2 & 3 \\
& Operating room & 8 & 20 & 28 \\
Total & - & 38 & 76 & 114
\end{tabular}

Note: Biological PCD, the number of sterilizers using biological $\mathrm{PCD}$ in daily monitoring for luminal devices. Common $\mathrm{BI}$, the number of sterilizers using common biological indicators in daily monitoring for luminal devices.

Abbreviations: PLADs=provincial-level administrative divisions; $\mathrm{PCD}=$ process challenge devices; $\mathrm{BI}=$ biological indicators; $\mathrm{CSSD}=$ central sterile supply department.

TABLE 2. Comparison of 338 times biological PCD monitoring and common biological monitoring of lowtemperature hydrogen peroxide sterilizers

\begin{tabular}{cccr}
\hline \multirow{2}{*}{ Common BI } & \multicolumn{2}{c}{ Biological PCD } & \multirow{2}{*}{ Total } \\
\cline { 2 - 3 } & Qualified & Unqualified & \\
\hline Qualified & 323 & 12 & 335 \\
Unqualified & 0 & 3 & 3 \\
Total & 323 & 15 & 338 \\
\hline
\end{tabular}

Note: Biological PCD, the number of sterilizers using biological $\mathrm{PCD}$ in daily monitoring for luminal devices. Common $\mathrm{BI}$, the number of sterilizers using common biological indicators in daily monitoring for luminal devices.

Abbreviations: $\mathrm{PCD}=$ process challenge devices; $\mathrm{BI}=$ biological indicators.

to assess the adequate performance of the process (2-4). According to the standard EN867, PCD is defined as an object, which simulates the worst case of conditions for the attainment of the specified sterilization conditions within the items to be sterilized (5). In our survey, the PCD contained a biological indicator and was placed in a position in which the sterilizer may have difficulty affecting. It should be noted that the PCD depends on the nature of the items to be sterilized.

The qualification rate of biological PCD was much lower compared to the common biological indicator, which can be interpreted as a biological luminal PCD device being more sensitive to the sterilization process. In this survey, $33.3 \%$ of the hospitals used biological PCD when sterilizing lumen devices, while the remaining hospitals were not aware of this problem.

When using hydrogen peroxide plasma sterilization, the items to be sterilized should be placed in the correct order and should not be covered $(6-7)$. 
However, in only $39.5 \%$ of the surveyed sterilizers, items to be sterilized were arranged in a single layer.

Hydrogen peroxide gas, which has a poor penetrating power, has a major role in sterilization progress. The sterilizing effect may be affected by the length, diameter, and material of the luminal devices (8-10), while common biological monitoring only reflects the sterilizing effects of device surfaces. In consideration of complex structures, the resistance of luminal devices to sterilization is much higher than in non-lumen devices. For these reasons, the application of luminal PCD can reflect relatively true sterilization effects. When sterilizing luminal devices, the routine use of biological luminal PCD is recommended so as to detect probable sterilization failures.

In this study standard biological PCD was used. However, under ideal conditions, the material, lumen length and inner diameter of biological PCDs should be consistent with the lumen devices which would more exactly reflect effective sterilization of lowtemperature hydrogen peroxide gas plasma sterilizers. The staff awareness of disinfection process monitoring needs to be included in further investigations.

Acknowledgments: The project members (22 provincial and municipal-level CDCs; Beijing Municipality, Tianjin Municipality, Hebei Province, Shanxi Province, Liaoning Province, Jilin Province, Heilongjiang Province, Shanghai Municipality, Jiangsu Province, Zhejiang Province, Anhui Province, Shandong Province, Henan Province, Hubei Province, Hunan Province, Guangdong Province, Sichuan Province, Guizhou Province, Yunnan Province, Gansu Province, Ningxia Hui Autonomous Region, Xinjiang Uygur Autonomous Region CDCs, Chinese People's Liberation Army CDC, and 58 sentinel hospitals).

Funding: The "National Surveillance Program for Hospital Disinfection and Sterilization Effects and Nosocomial Infection”.

doi: $10.46234 / \mathrm{ccdcw} 2021.152$

\# Corresponding author: Jin Shen, shenjin@nieh.chinacdc.cn.
${ }^{1}$ National Institute of Environmental Health, Chinese Center for
Disease Control and Prevention, Beijing, China.

Submitted: December 08, 2020; Accepted: June 30, 2021

\section{REFERENCES}

1. Zhang J, Xing SX, Qiu X, Wang LF, Zhang LB. PCD used in monitoring sterilization efficacy of hydrogen peroxide gas plasma sterilizer. Chin J Nosocomiol 2009;19(12):1531 - 3. http://dx.doi.org/ 10.3321/j.issn:1005-4529.2009.12.022.

2. AAMI. AAMI TIR31: 2003 Process challenge devices/test packs for use in health care facilities. Arlington, VA: Association for the Advancement of Medical Instrumentation, 2003. https://store.aami.org/s/store\#/ store/browse/detail/a152E000006j609QAA.

3. State Administration for Market Regulation, Standardization Administration. GB 27955-2020 Hygienic requirements for lowtemperature hydrogen peroxide gas plasma sterilizer. Beijing: China Standard Press, 2020. (In Chinese).

4. Ban HQ. Disinfection monitoring technology guide in health care. Zhengzhou: Zhengzhou University Press. 2017: 115-8. (In Chinese).

5. Standards Policy and Strategy Committee. EN 867-5: 2001 Nonbiological systems for use in sterilizers-Part 5: specification for indicator systems and process challenge device for use in performance testing for small sterilizers Type B and Type S. European Committee for Standardization, 2001. https:/www.en-standard.eu/csn-en-867-5-nonbiological-systems-for-use-in-sterilizers-part-5-specification-forindicator-systems-and-process-challenge-devices-for-use-inperformance-testing-for-small-sterilizers-type-b-and-type-s/.

6. You N, Yao Y, Zhang X. Cycle mode and effect evaluation method of hydrogen peroxide plasma sterilization. Chin J Nosocomiol 2017;27(3):705 - 9. http://dx.doi.org/10.11816/cn.ni.2016-162940. (In Chinese).

7. Ding ZM, Gao XN, Yu YQ, Zou YF, Zhang Y, Wang JM, et al. Management of quality of sterilization with hydrogen peroxide low temperature plasma. Chin J Nosocomiol 2014;24(24):6238-40. http://dx.doi.org/10.11816/cn.ni.2014-144192. (In Chinese).

8. Unger-Bimczok B, Kosian T, Kottke V, Hertel C, Rauschnabel J. Hydrogen peroxide vapor penetration into small cavities during lowtemperature decontamination cycles. J Pharm Innov 2011;6(1):32 - 46. http://dx.doi.org/10.1007/s12247-011-9097-3.

9. Kanemitsu K, Imasaka T, Ishikawa S, Kunishima H, Harigae H, Ueno $\mathrm{K}$, et al. A comparative study of ethylene oxide gas, hydrogen peroxide gas plasma, and low-temperature steam formaldehyde sterilization. Infect Control Hosp Epidemiol 2005;26(5):486-9. http://dx. doi.org/10.1086/502572.

10. Rutala WA, Gergen MF, Weber DJ. Comparative evaluation of the sporicidal activity of new low-temperature sterilization technologies: ethylene oxide, 2 plasma sterilization systems, and liquid peracetic acid. Am J Infect Control 1998;26(4):393 - 8. http://dx.doi.org/10.1016/ S0196-6553(98)70034-3. 